\title{
Epineurial pseudocyst of the facial nerve: prevalence and clinics
}

\author{
Laura Celis ${ }^{1,2}$ (D) Bert De Foer ${ }^{3}$ (D) Frank Declau ${ }^{1,2}$ (D), Laura Pingnet ${ }^{1,4}$ (D), Anja Bernaerts ${ }^{3}$ (D), Dirk Vanden Abeele ${ }^{1}$ \\ 1'Department of Otorhinolaryngology, GZA-hospitals, Campus Sint-Vincentius, Antwerp, Belgium \\ ${ }^{2}$ University of Antwerp, Faculty of Medicine and Health Sciences, Antwerp, Belgium \\ ${ }^{3}$ Department of Radiology, GZA-hospitals, Antwerp, Belgium \\ ${ }^{4}$ Department of Otorhinolaryngology, Universital hospital Antwerp, Edegem, Belgium
}

Cite this article as: Celis L, De Foer B, Declau F, Pingnet L, Bernaerts A, Vanden Abeele D. Epineurial pseudocyst of the facial nerve: prevalence and clinics. B-ENT 2020; 16(2): 109-14.

\begin{abstract}
Objective: Epineurial pseudocysts (EPs) of the facial nerve are considered uncommon, benign lesions originating from and in close correlation with the mastoidal segment of the facial nerve. The objective of this study was (i) to investigate the prevalence of EP in a large study population undergoing a cone-beam computed tomography (CBCT) of the temporal bone for various neurotological reasons, (ii) to discuss the imaging features of EP, and (iii) to evaluate a potential correlation between the EP of the facial nerve and clinical neurotological symptoms.

Methods: A retrospective review of 559 CBCTs of the temporal bone was performed to investigate the occurrence of EP. Radiological characteristics, demographic information, relevant medical history, neurotological symptoms, and findings from the EP positive cases were analyzed.

Results: The prevalence of EP was $1.61 \%$. Characteristic radiological features of EP included a sharply delineated, round to oval structure with soft tissue density in proximity and posterior to the mastoidal segment of the facial nerve. Although in close relation to the facial nerve, no association with facial motor signs was observed.

Conclusion: EP cannot be regarded as an infrequent lesion. EP is an incidental finding in which clinical findings and complaints appear to be unrelated to the presence of EP.
\end{abstract}

Keywords: Computed tomography, epineurial pseudocyst, facial nerve, prevalence,

temporal bone

\section{Introduction}

Epineurial pseudocysts (EPs) of the facial nerve are considered benign lesions originating from and in close correlation with the mastoidal segment of the facial nerve. To the best of our knowledge, only two studies were published on this type of lesion $(1,2)$. A case report of an incidental finding of an EP, which was discussed at the annual spring meeting of the Royal Belgian Society for Ear, Nose Throat, Head and Neck Surgery in 2019, drew our attention to his rather unknown entity (3). These lesions are reported to be uncommon and are mostly incidental findings encountered during imaging of the temporal bone or during middle ear surgery. The paucity of literature on EPs may explain why most of these lesions are not appreciated preoperatively.
Histopathology, as described by Pertzborn et al. (1), revealed an amorphous stroma with a dense fibrovascular tissue lacking a true cyst wall. Neither malignancy nor nervous tissue was identified. Owing to the above-mentioned characteristics, the entity was named EP.

Information is currently lacking on prevalence, natural history, clinical significance, and the necessity for further investigation or further treatment of these lesions. The primary goal of this study was to determine the prevalence of EP in patients undergoing a cone beam (CB) computed tomography (CT) (CBCT) of the temporal bone due to various neurotological symptoms. In addition, patient characteristics and neurotological symptoms were reviewed to investigate a potential association with EP.

Corresponding Author: Laura Celis, celis.laura@outlook.com

Received: July 12, 2020 Accepted: December 14, 2020

Available online at www.b-ent.be

CC BY 4.0: Copyright@Author(s), "Content of this journal is licensed under a Creative Commons Attribution 4.0 International License." 

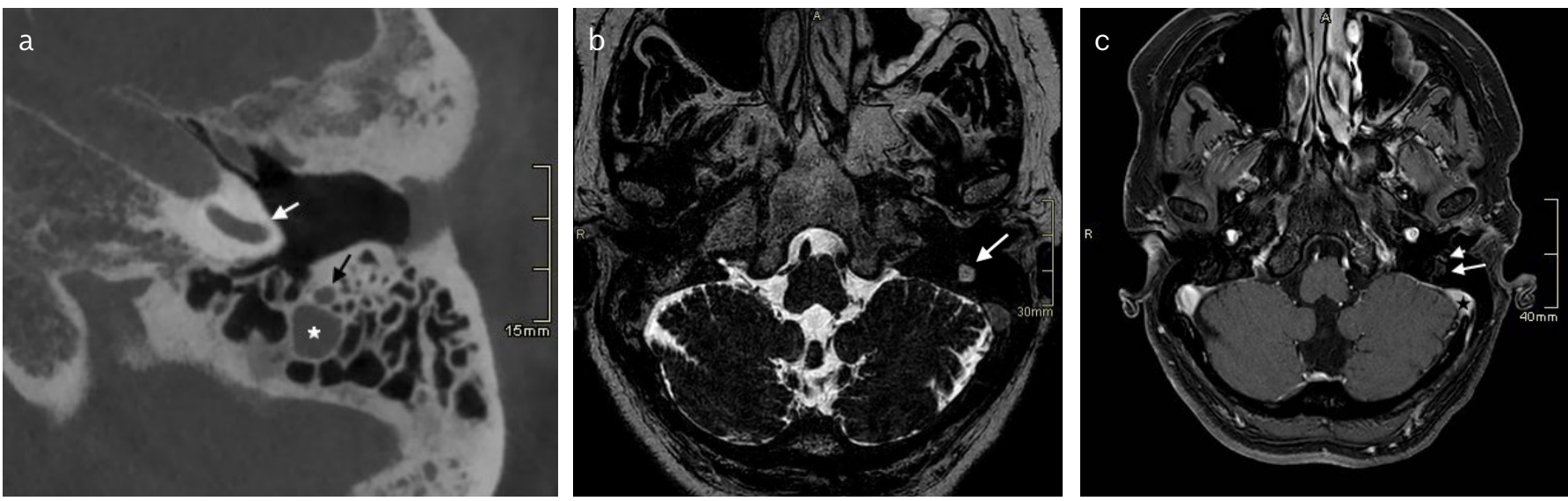

Figure 1. a-c. Case 4. Axial cone-beam computed tomography (CBCT) image at the level of the basal turn of the cochlea (white arrow). Note the oval, sharply delineated punched-out soft tissue lesion (asterisk) located immediately posterior to the mastoid segment of the facial nerve on the left side (black arrow). Note the close relationship of the lesion to the mastoidal segment of the facial nerve (a). Axial submillimeter three-dimensional (3D) Turbo Spin-Echo T2-weighted magnetic resonance imaging (MRI) through the lower skull base showing the moderate intense roundish lesion on the left side (arrow) (b). Gadolinium enhanced 3D gradient-echo T1-weighted MRI at the level of the sigmoid sinus (asterisk). Note the small hypointense nodule (arrow) with some subtle peripheral enhancement located immediately posterior to the enhancing mastoidal segment of the facial nerve (arrowhead) (c).

\section{Methods}

A retrospective study was set up in a single hospital setting to investigate the prevalence of the EP of the mastoidal segment of the facial nerve. All CBCTs of the temporal bone in patients referred by our Ear Nose Throat (ENT) department between January 2015 and September 2019 were reviewed. The initial indications for neurotological evaluation by CBCT imaging included hearing loss, otitis media, tinnitus, aural fullness, and vertigo. Imaging data were collected from the Picture Archive and Communication System (PACS) of the department of radiology. In total, 559 patients were included. Imaging evaluation was performed either on a multidetector $\mathrm{CT}$ or on a CBCT of the temporal bone using submillimeter slices in an ul-

\section{Main Points:}

Epineurial pseudocysts (EPs) of the facial nerve are considered benign lesions originating from and in close correlation with the mastoidal segment of the facial nerve.

Characteristic radiological features of EP at cone-beam computed tomography of the temporal bone include a sharply delineated, round to oval structure with soft tissue density in proximity and posterior to the mastoidal segment of the facial nerve.

Although a rather unknown entity, EP cannot be regarded as an infrequent lesion with a prevalence of $1.61 \%$ in our study population

On the basis of our results, we regard EP as an incidental finding in which clinical findings and complaints seem to be unrelated to the presence of EP. Although in close relationship to the facial nerve, no association with facial motor signs was observed in our series.

Because they do not seem to grow, EPs should be considered as a do-not-touch lesion in the case of absence of facial nerve symptomatology. Knowledge of the entity is required for both Ear, Nose, and Throat surgeons and radiologists because the location of these lesions might influence the surgical approach in the case of mastoid surgery. tra-high-resolution bone algorithm with axial and coronal reformations in a small field for a view $(6 \mathrm{~cm})$ centered over each temporal bone individually. All the 559 CBCTs of the temporal bone were independently evaluated by two physicians: a senior ENT resident and a senior staff member in radiology with 25 years of experience in head and neck radiology. In case of disagreement, a consensus was reached by joint reading. Analysis of the temporal bone CBCT was performed on the PACS system monitors using the axial and coronal reformations in bone window for each ear separately $(n=1,118)$. The typical characteristics of the EP that were sought for included a sharply demarcated, expansile structure with soft tissue density without any clear cortical bone density delineation. The lesion was contiguous with and located posterior to the mastoid portion of the facial nerve canal, mimicking the appearance of an isolated opacified mastoid air cell in close contact with the mastoidal segment of the facial nerve (Figure 1. a). Exclusion criteria included severe mastoid opacification or a medical history of middle ear surgery by mastoidectomy.

Demographic information on the patients with EP was retrospectively reviewed. In addition, relevant medical history, clinical presentation, findings by neurotological examination from the patient's medical files, and imaging features on magnetic resonance imaging (MRI), if available, were included in the analysis. To investigate the association between clinical findings and EP, Chi-square analysis was used. Wilcoxon matched-pair signed-rank test was used to study the relationship between EP and the Pure Tone Average (PTA).

This study was approved by the Ethics Committee for Clinical Investigations (decision number: 200305RETRO). The need for informed consent was waived owing to the retrospective character of the study.

\section{Results}

\section{Radiological Findings}

CBCTs of the temporal bone of 559 patients were evaluated, including a total of 1,118 individual ears. One hundred and thir- 
Table 1. Patient characteristics

\begin{tabular}{|c|c|c|c|c|c|c|c|c|c|}
\hline Patient & Age/Sex & Lesion & Medical History & Micro-otoscopy & $\begin{array}{l}\text { Hearing loss, } \\
\text { PTA }\end{array}$ & $\begin{array}{l}\text { Facial } \\
\text { nerve palsy }\end{array}$ & Tinnitus & $\begin{array}{l}\text { Aural } \\
\text { pressure }\end{array}$ & Vertigo \\
\hline 1 & $30 / F$ & Right & - & $\begin{array}{l}\text { Pars flaccida } \\
\text { cholesteatoma } \\
\text { right }\end{array}$ & $\begin{array}{l}\mathrm{CHL} \text { right, } \\
43 \mathrm{~dB} \mathrm{HL}\end{array}$ & - & Right & - & - \\
\hline 2 & $38 / F$ & Right & - & Normal & $\begin{array}{l}\text { SNHL right, } \\
32 \mathrm{~dB} \mathrm{HL}\end{array}$ & - & Right & Right & - \\
\hline 4 & $60 / F$ & Left & VCN right & Normal & $\begin{array}{l}\text { SNHL right, } \\
33 \mathrm{~dB} \mathrm{HL}\end{array}$ & - & - & Right & $\begin{array}{l}\text { BPPV left, } \\
\text { areflexia } \\
\text { right }\end{array}$ \\
\hline 7 & $53 / \mathrm{M}$ & Left & $\begin{array}{l}\text { Cholesteatoma } \\
\text { surgery right }\end{array}$ & $\begin{array}{l}\text { Pars flaccida } \\
\text { cholesteatoma } \\
\text { right }\end{array}$ & $\begin{array}{l}\mathrm{CHL} \text { right, } \\
47 \mathrm{~dB} \mathrm{HL}\end{array}$ & - & - & - & - \\
\hline 8 & $52 / \mathrm{M}$ & Left & - & Normal & $\begin{array}{l}\text { CHL left, } \\
\text { 30dB HL }\end{array}$ & - & - & Left & - \\
\hline 9 & $12 / F$ & Bilateral & - & Normal & $\begin{array}{l}\text { CHL left, } \\
37 \mathrm{~dB} \mathrm{HL}\end{array}$ & - & - & - & - \\
\hline 10 & $32 / F$ & Bilateral & $\begin{array}{l}\text { Turner } \\
\text { syndrome }\end{array}$ & $\begin{array}{l}\text { Tympanic } \\
\text { perforation right }\end{array}$ & $\begin{array}{l}\text { SNHL } \\
\text { bilateral, } \\
\text { 30dB HL }\end{array}$ & - & - & - & - \\
\hline
\end{tabular}

BPPV: Benign paroxysmal positional vertigo; CHL: Conductive hearing loss; F: Female; HL: Hearing loss; M: Male; PTA: Pure tone average; SNHL: Sensorineural hearing loss; SSNHL: Sudden sensorineural hearing loss; VCN: Vestibulocochlear neurinoma

ty-seven ears were excluded because of severe mastoid opacification $(n=77)$ or previous middle ear surgery by mastoidectomy $(n=60)$. The less experienced physician (senior ENT resident) identified 10 ears with imaging features matching those of an EP of the facial nerve. The more experienced physician (senior head and neck radiologist) identified an additional six EPs besides those mentioned above. After a comparison of the results of the two observers, a consensus was reached to ultimately include 12 patients-16 ears-showing EPs, four out of which with bilateral EPs, three with a right-sided EP, and five with a left-sided EP. The prevalence of EP was $1.63 \%$ (16 on 981 ears).

A retrospective review of the original radiological report of the CBCT examination of the temporal bone of the included EP cases was positive in only one case for a suspicious finding in or near the mastoidal segment of the facial nerve.

Observed characteristics of EP on CBCT included a sharply delineated, punched out round to oval structure with soft tissue density in proximity and posterior to the mastoidal segment of the facial nerve. The size was small but variable $(1.2-7.3 \mathrm{~mm})$. The lesion showed a typical configuration; it was located posterior to and abutting the mastoid portion of the facial canal in all cases. The facial nerve canal was not dehiscent. The EP often mimicked the appearance of an isolated opacified mastoid air cell (Figure 1a).

MRI was performed in two patients, which confirmed the presence of a roundish lesion with moderate to high signal on T2-weighted sequences (Figure 1b) and low signal on T1-weighted sequences located low and posterior into the signal void of the temporal bone. After intravenous gadolinium administration, subtle rim enhancement was observed (Figure 1c). Non-Echo-Planar Diffusion-weighted MRI (Non-EPI DW MRI) showed no hyperintensity on b1000 images, differentiating it from a cholesteatoma in one case (4).

Previous imaging data or repeated imaging studies were available in three patients, up to a maximum of six years follow-up. 
On repeat $\mathrm{CBCT}$, the characteristics of the EP were noted to remain unchanged in all three cases, and no appreciable growth was recorded.

\section{Patient Demographics}

Of the 12 included patients, the male to female ratio was 5:7. The mean age was 45 years (range, 12-81 years). One unilateral case presented a coincidental finding of a vestibulocochlear schwannoma on the contralateral side. One bilateral case showed a congenital malformation of the right-sided external ear canal and pinna and a history of reconstructive surgery of the auricle. One case was known to have Turner syndrome.

\section{Clinical Findings}

The clinical presentation of the patients with EP is summarized in Table 1.

Patients presented with various neurotological symptoms leading to the indication of CBCT of the temporal bone. Most common were hearing loss $(n=12)$, aural fullness $(n=4)$, tinnitus $(n=4)$, otalgia $(n=3)$, and vertigo $(n=2)$.

None of the patients had a history of facial nerve symptoms. Neither current nor previous episodes of facial palsy were documented. No facial weakness, facial spasticity, or facial pain was registered. Clinical examination of the facial nerve function in all the patients was normal (House-Brackmann classification Grade 1). Because of the lack of clinical signs of facial nerve dysfunction, Electroneuronography (ENOG) or electromyography (EMG) of the facial nerve were not performed.

All patients presented with hearing loss. Within the group of unilateral EPs $(n=8)$, three patients presented with unilateral conductive hearing loss, two out of which were on the ipsilateral side regarding the EP ear and one on the contralateral side. Sensorineural hearing loss was documented in five patients in the unilateral EP group $(n=8)$, three in the ipsilateral, and one in the contralateral regarding the side of the EP. Another patient with unilateral EP showed presbyacusis with bilateral symmetrical high-frequency sensorineural hearing loss.

Within the group of bilateral EPs $(n=4)$, three patients presented with unilateral conductive hearing loss. One patient presented with bilateral sensorineural hearing loss. A comparison of the PTA revealed no difference (Wilcoxon matched-pair signed-rank test) between the ipsilateral EP side and contralateral EP side.

Clinical examination by micro-otoscopy revealed a pars flaccida cholesteatoma in two patients with unilateral conductive hearing loss: one in the contralateral ear and one in the ipsilateral ear regarding the EP side. After diagnostic evaluation and imaging, they both underwent middle ear surgery by simple mastoidectomy, tympanoplasty, and reconstruction of the ossicular chain. Although the EP was not preoperatively encountered, no peroperative or postoperative complication such as facial nerve palsy was observed. Another patient presented with a tympanic perforation causing the conductive hearing loss.

Four patients mentioned unilateral tinnitus at their EP side, all of which were associated with hearing loss on audiometry (discrete low-frequency conductive hearing loss in one patient and a high-frequency perceptive hearing loss in three other patients)
Aural fullness was registered in four patients of the unilateral EP group. Three patients experienced aural pressure in the ipsilateral ear, one in the contralateral ear, but tympanometry showed normal compliance of the middle ear in all the cases.

Dizziness was mentioned by two patients. One suffered from rotatory vertigo and was diagnosed with benign paroxysmal positional vertigo (BPPV) at the EP side and a vestibular areflexia on the contralateral side owing to the vestibular schwannoma. Another patient experienced instability; electronystagmography revealed a centrally compensated vestibular areflexia on the EP side.

There was no association between the occurrence of the clinical symptoms aural pressure, tinnitus, and vertigo and the side of EP (Chi-square $p=0.52)$, suggesting no link between the EP and these symptoms.

\section{Discussion}

First described in 2003 by Pertzborn et al (1), EP of the facial nerve is regarded as an uncommon entity. The paper described a case series of seven EPs, all of which had been encountered in association with the facial nerve intraoperatively (4 of 7 ) or with preoperative CT imaging (3 of 7 ). In the initial series, all of the patients underwent a tympanomastoidectomy: four for cochlear implantation because of profound sensorineural hearing loss and three for facial nerve symptoms such as facial spasticity, recurrent facial palsy, or facial pain. A postoperative review of all the seven preoperative $\mathrm{CT}$ scans demonstrated radiographic abnormalities abutting the facial canal with the known characteristic features. All CT scans showed a sharply delineated, non-enhancing soft tissue lesion adjacent to the mastoidal segment of the facial nerve. Lesions seemed to be contiguous to the mastoidal portion of the facial nerve canal. The facial nerve canal was often dehiscent (5 of 7). Macroscopic evaluation of all lesions during middle ear surgery revealed a soft compressible, balloon-like, pedunculated lesion with a fibrous capsule in continuity with the epineurium of the facial nerve. Histopathology of the lesions was consistent with dense fibrovascular tissue. None of the specimens revealed malignancies or nervous tissue, ruling out schwannomas and neuromas. In addition, no epithelium or true cyst was identified, ruling out the differential diagnoses of mucosal disease of a mastoid air cell (1).

Although pseudocysts of the facial nerve were likely no novel findings, they had not been clearly described in the literature before 2003. Following Pertzborn's publication on the entity of EP, just a few papers discussing the differential diagnoses of facial nerve tumors referred to EPs (5-8). In addition, no further research was published on this topic until recently by the group of Delrue who investigated a case series of 10 patients in parallel to our ongoing study (2). All patients diagnosed with EPs of the intratemporal facial nerve during a period of nine years were retrospectively reviewed. The latter study demonstrated that $50 \%$ of the patients exhibited bilateral EPs, which is a higher bilateral frequency compared with our series $(33.3 \%$ bilateral). The mean age was 35.7 years. In our series, the mean age was 45 years. Within their series, facial nerve dysfunction was not observed, which is in agreement with our results. In addition, the lesions were incidental findings and did not re- 
quire further surgical exploration, resulting in a lack of additional histological information, which is also in accordance with our findings.

Data regarding the prevalence of EP are non-existent because prevalence has not been investigated. In our study, we reached a consensus on diagnosing 16 EPs out of 981 included ears, resulting in a prevalence of $1.63 \%$. The prevalence threshold that defines a rare disease varies across jurisdictions and organizations, ranging from 5 to 76 cases per 100,000 individuals in the general population (9). On the basis of this definition, our results show a higher prevalence within the sampled population and do not fit the definition of a rare disease. However, interpreting this data, we must consider the retrospective nature of this single hospital study and a potential selection bias: all of the patients were referred for imaging by the ENT surgeon and presented with neurotological symptoms. If EP was associated with neurotological symptoms or (middle) ear pathology, the prevalence within our sampled population would be higher compared with that of the general population.

As mentioned earlier, all included patients diagnosed with EP exhibited a variety of clinical symptoms for which imaging was performed, ranging from hearing loss to otalgia, aural fullness, tinnitus, and vertigo. These symptoms were observed contralaterally and ipsilaterally in the same ratio, regardless of the EP side. In most cases, symptoms were most likely related to non-EP-related pathology such as cholesteatoma, tympanic membrane perforation, otitis externa, vestibular schwannoma, BPPV, on the basis of Chi-square analysis and the Wilcoxon test, there is no association between EP and ipsilateral neurotological symptoms, suggesting no link between the EP and these symptoms. In addition, the incidence of a contralateral vestibulocochlear schwannoma (Case 4), the history of Turner syndrome (Case 10), and unilateral congenital malformation of the outer ear in Case 12 with bilateral EPs are most likely coincidental comorbidity and not related to the EP.

In contradiction to the previous observation of Pertzborn but in line with the series of Delrue, none of our included cases demonstrated history of facial nerve symptoms. In the original series, three out of seven patients experienced facial nerve symptoms for which further diagnostic imaging was performed, leading to the diagnosis of EP (1). Although they could not conclude that the EPs were responsible for the facial motor signs and symptoms, they considered a potential role of EP in facial nerve dysfunction. Dissection of the EP in those patients improved their facial nerve symptoms partially, with no recurrence of facial spasticity nor facial palsy episodes. Because of the lack of facial nerve symptoms in our study, no further investigation of facial nerve function by ENOG or EMG was performed. Therefore, no final conclusion can be made whether EP interferes with subclinical facial nerve function, but the lack of any symptoms of facial nerve dysfunction in our study population is a striking feature and is opposed to the results of Pertzborn. On the basis of the above results, in the majority of cases, EP can be regarded as an incidental finding.

This study demonstrates that these lesions are generally underdiagnosed. Initially, only one out of the 16 included EPs was recognized and correctly assigned as EP in the original report of the radiologist. When the same imaging studies were re- viewed in this study by a targeted EP screening, a total of 16 cases were withheld by the more experienced physician and a total of 10 cases by the less experienced physician. As a first conclusion, the lack of familiarity with EP and the absence of a targeted search resulted in a significantly lower number of identified cases. This can be explained by the paucity of literature on EPs. Knowledge and awareness of EP obviously result in a higher detection rate. As a second conclusion, general clinical experience clearly facilitates the recognition of Eps because the more experienced physician confirmed almost twice as many cases. Other factors complicating the recognition of EP include the presence of chronic otitis media because severe mastoidal opacification impedes proper differential diagnosis between EP and an opacificated mastoidal cell, resulting in the exclusion of 77 ears in this study. It is possible that a number of EPs might have been overlooked in these excluded cases.

Furthermore, differential diagnosis of EP with other pathologies can be challenging but possible in most cases owing to specific radiographic features. The differential diagnosis for lesions along the mastoid segment of the facial nerve include inflammatory changes, cholesteatomas, and (intrinsic) facial nerve tumors $(5,6)$. Inflammatory changes (otitis media) would likely involve more mastoid cells. Acquired cholesteatomas usually originate in another location and are more frequently located in the middle ear in case of a pars flaccida cholesteatoma. Pars tensa cholesteatoma often involves the inferior and posterior part of the tympanic membrane and posterior hypotympanon. However, they do not demonstrate such an intimate relationship with the facial nerve. Congenital cholesteatoma can involve the facial nerve canal but is more frequently located near the region of the geniculate ganglion. Acquired as well as congenital cholesteatoma can be confirmed or ruled out by using non-EPI DWI MRI (4). The most common intrinsic tumors of the facial nerve are schwannomas, followed by hemangiomas or venous vascular malformation of the facial nerve, but a variety of other lesions have been reported in the literature, including meningioma, neurofibroma, angioma, paraganglioma, chondromyxoid fibroma, rhabdomyoma, and granular cell tumors (5). The description of the differential diagnosis of these lesions is beyond the scope of this paper, but we will highlight the differential diagnostic features of the most important ones. Schwannoma has by definition a central location relative to the facial nerve course, in contradiction to EP, demonstrating a more eccentric pattern. Schwannomas can have a growth pattern outside of the facial nerve canal but even then, they usually grow anteriorly into the middle ear cavity in case they originate from the mastoid segment of the facial nerve. Hemangiomas or venous vascular malformation of the facial nerve can be differentiated by the fact that they are situated in the geniculate ganglion region and that they display dot-like calcifications in the lesion on CBCT. Moreover, they will enhance after intravenous Gadolinium administration, discriminating them from EPs. Hence, the aspect, location, and imaging features of EPs can be regarded as fairly typical.

The underlying pathogenesis of EP is currently unknown. A potential hypothesis is chronic exposure of the facial nerve to an inflammatory process in the mastoid cavity, causing proliferation of fibrovascular tissue. Dehiscence of the facial nerve canal was observed in several cases in the initial series (1), backing up 
this hypothesis. However, no facial nerve canal dehiscence was observed in our series.

Although often underdiagnosed, preoperative recognition of EP by the ENT surgeon is crucial because the presence of EP can have implications for certain surgical approaches. The presence of a large EP in the facial recess or on the lateral aspect of the mastoid segment of the facial nerve may impact the placement of a cochlear implant electrode array or completion of a modified radical mastoidectomy. In one patient diagnosed with a cholesteatoma on the EP side, a mastoidectomy was performed without preoperative awareness of the EP. Even so, no abnormality was encountered during surgery; the procedure was completed, and no postoperative complications such as facial palsy were observed.

Because the literature on EP is currently extremely limited, the natural history of this lesion is unknown. However, EPs are considered to be benign and stable lesions. This was first stated by Pertzborn who reviewed one patient subjected to a control CT after four years before resection of the lesion. During this period of follow-up, no growth or change of the lesion could be observed. In our series, we observed similar findings. On consecutive CTs in three patients, the EP was unchanged, and no appreciable growth was noted up to a maximal follow-up period of six years. Consequently, it is likely that in the absence of facial nerve symptoms, these lesions can be considered as incidental findings and do not require surgical intervention at all. However, in the case of facial nerve symptoms, surgical exploration can be proposed because in the initial case series of Pertzborn, all the seven EPs were resected successfully with preservation of the anatomy and function of the facial nerve (1). However, this could not be confirmed in our series.

Limitations of this study include the relatively small number of EP cases. Hence, an even larger study population should be analyzed in the future to increase the number of EP cases, thereby allowing more insight into the association of patient symptoms and characteristics and the presence of EP. Furthermore, no details regarding macroscopic peroperative findings or histology are available because resection of EP lesions was not executed in our confirmed subjects. Therefore, it is unknown whether these cases, in analogy with those of Pertzborn, could be resected safely, would respond to neurostimulation, were in continuity with the epineurium, and did not contain neural tissue.

In conclusion, our study proves that EP cannot be regarded as an infrequent lesion with a prevalence of $1.61 \%$ in our study population. On the basis of our results, we regard EP as an incidental finding in which clinical findings and complaints seem to be unrelated to the presence of EP. Because they also do not seem to grow, EPs should be considered as do-not-touch lesions in the case of absence of facial nerve symptomatology. Knowledge of the entity is required for both ENT surgeons and radiologists because the location of these lesions might influence surgical approaches in cases of mastoid surgery.

Ethics Committee Approval: This study was approved by the Ethics Committee for Clinical Investigations (Approval No: 200305RETRO).

Informed Consent: Informed consent is not necessary due to the retrospective nature of this study.

Peer-review: Externally peer-reviewed.

Author Contributions: Supervision - B.D.F., DV.A., F.D.; Design D.V.A., L.C.; Resources - P.R., C.H., H.R.; Materials - L.C., B.D.F., D.V.A., F.D., A.B.; Data Collection and/or Processing - L.C., B.D.F., D.V.A., F.D., A.B.; Analysis and/or Interpretation - L.C., B.D.F., D.V.A., F.D., A.B.; Literature Search - L.C., L.P.; Writing Manuscript - L.C.; Critical Review - L.P., B.D.F., D.V.A., F.D., A.B.

Conflict of Interest: The authors have no conflict of interest to declare.

Financial Disclosure: The authors declared that this study has received no financial support.

\section{References}

1. Pertzborn SL, Reith JD, Mancuso AA, Antonelli PJ. Epineurial pseudocysts of the intratemporal facial nerve. Otol Neurotol 2003; 24: 490-3. [Crossref]

2. Delrue S, Cammaert T, Heylbroeck P, Lemmerling M. Epineurial pseudocyst of the intratemporal facial nerve: A case series study. J Int Adv Otol 2020; 16: 266-70. [Crossref]

3. Pingnet L, Vanden Abeele D. Epineurial pseudocyst of the intratemporal facial nerve: Report of a rare case and review of the literature. B-ENT 2019;15: 41.

4. De Foer B, Vercruysse JP, Bernaerts A, et al. Middle ear cholesteatoma: Non-echo-planar diffusion-weighted MR imaging versus delayed gadolinium-enhanced T1-weighted MR imaging--value in detection. Radiology 2010; 255: 866-72. [Crossref]

5. Prasad SC, Laus $M$, Dandinarasaiah $M$, et al. Surgical management of Intrinsic tumors of the facial nerve. Neurosurgery 2018; 83:740-52. [Crossref]

6. Veillon F, Taboada LR, Eid MA, et al. Pathology of the facial nerve. Neuroimaging Clin N Am 2008; 18: 309-20. [Crossref]

7. Borges A. Pathology of the facial nerve. Lemmerling M, De Foer B, editors. Temporal Bone Imaging. Berlin, Germany: Springer-Verlag; 2015.p.285.

8. Michalopoulos K, Bajaj Y, Strachan DR. Recurrent facial nerve palsy caused by a facial cyst. Br J Hosp Med 2008; 69: 475. [Crossref]

9. Richter T, Nestler-Parr S, Babela R, et al. Rare disease terminology and definitions-a systematic global review: Report of the ISPOR rare disease special Interest group. Value Health 2015; 18: 906-14. [Crossref] 